ROCZNIKI HUMANISTYCZNE

Tom LXVIII, zeszyt 5 - 2020

DOI: http://dx.doi.org/10.18290/rh20685-15

REBECA LÁZARO NISO

\author{
LECTURAS Y REESCRITURAS \\ DE HECHOS DE BERNARDO DEL CARPIO
}

\begin{abstract}
R e s u m e n. A lo largo del Siglo de Oro la reescritura de obras dramáticas se convierte en una forma común de creación literaria. Pocos son los dramaturgos que no sintieron la necesidad de volver sobre las obras de sus predecesores con la intención de mejorar o actualizar textos frente a los gustos cambiantes del público. Este fenómeno general se ejemplifica en este trabajo a través de la reescritura en dos versiones impresas distintas llevada a cabo por Álvaro Cubillo de Aragón de una obra suya dedicada a la materia histórico-legendaria relacionada con la figura de Bernardo del Carpio: El conde de Saldaña y hechos de Bernardo del Carpio.
\end{abstract}

Palabras clave: Siglo de Oro; reescritura; obras dramáticas; Bernardo del Carpio; Cubillo de Aragón; Hechos de Bernardo del Carpio.

La figura de Bernardo del Carpio ha conocido un cultivo constante en la literatura española y mantuvo, por supuesto, una presencia duradera en la tradición teatral del Siglo de Oro donde se afianzó el mito de su figura, convirtiéndolo en el arquetipo de héroe épico hispano, y se adornó con los atributos de los modelos caballerescos de Ariosto (Chevalier 209). La recurrencia a la materia legendaria de Bernardo despertó tal interés en los dramaturgos áureos, que fueron varias las comedias en las que el paladín español cobró protagonismo y otras tantas en las que ocupó un lugar preeminente (ver Ratcliffe 521-526 y Lázaro 79-95). El primer dramaturgo en ocuparse de la figura de Bernardo del Carpio fue Juan de la Cueva, introductor de la materia histórica en el teatro español, que en 1588 escribió Comedia de la libertad de España por Bernardo del Carpio (ver Watson). Cervantes también se

Dra. REBECA LÁZARO NiSO - profesora contratada interina, Universidad de La Rioja, Facultad de Letras y de la Educación, Departamento de Filologías Hispánica y Clásica; dirección para correspondencia: C/ San José de Calasanz, 33, 26004 Logroño, La Rioja, España; correo electrónico: rebeca.lazaro@unirioja.es. ORCID: https://orcid.org/00000001-8227-5304. 
dejó seducir por la figura del paladín hispano, que aparece citado en repetidas ocasiones en el Quijote (ver Gómez Redondo 1295-1298) y, aunque no pudo llevarlo a cabo, tenía en mente dedicarle una obra completa como aseguró en la dedicatoria del Persiles. ${ }^{1}$ En la materia que analizamos escribió Cervantes La casa de los celos y selvas de Ardenia, quizá la más denostada de toda su producción dramática. En este sentido, Menéndez Pelayo la consideró uno de los «solemnes desatinos» teatrales de Cervantes, señalando que se trataba de «una serie de visiones estrafalarias e inconexas» (Menéndez Pelayo 119-120). A pesar de ello, Rey Hazas y Sevilla Arroyo han hecho recientes intentos de rehabilitarla críticamente: «es una comedia perfectamente coherente, de sentido metaliterario, que pone en solfa las tradiciones caballerescas y pastoriles que utiliza, las parodia y se ríe de ellas» (Ver Rey Hazas y Sevilla Arroyo 1958-1966). Lope de Vega, por su parte, dedicó varias de sus obras a la figura de Bernardo. Según la Cronología de Morley-Bruerton, en primer lugar, escribió El casamiento en la muerte en 1597 y después Las mocedades de Bernardo del Carpio (1599-1608) (Morley y Bruerton 515 y Antonucci 71). Hay que mencionar también una comedia que llevaba por título Bernardo del Carpio. Segunda parte, que Cotarelo, valiéndose del encabezamiento de la pieza como Segunda parte y de los versos finales en la que «su autor os convida y llama / a la tercera comedia», ${ }^{2}$ atribuyó a Lope, aunque tal atribución fue después desmentida por Morley y Bruerton. En dicha obra Bernardo viaja a Italia acompañado de Roldán para ofrecer su ayuda al Papa e impedir a Teodosio que invada Italia. De nuevo, el perfil del héroe dista mucho del paladín legendario. Cubillo de Aragón, por su parte, dedicó a la materia histórico-legendaria relacionada con la figura de Bernardo del Carpio un drama en dos partes: El conde de Saldaña y El conde de Saldaña y hechos de Bernardo del Carpio. Curiosamente, tanto de la primera como de la segunda parte, contamos con dos versiones distintas, la primera con una transmisión manuscrita y la segunda a través de una tradición impresa. La comedia manuscrita de la primera parte lleva por nombre El bastardo de Castilla, mientras que su versión impresa El conde de Saldaña. Al contrario sucede con la segunda parte que tanto en la versión impresa como la manuscrita llevan el mismo nombre El conde de Saldaña y hechos de Bernardo del Carpio.

\footnotetext{
${ }^{1}$ «Todavía me quedan en el alma ciertas reliquias y asomos de Las semanas del jardín y del famoso Bernardo. Si a dicha, por buena ventura mía, que ya no sería ventura, sino milagro, me diese el cielo vida, las verá, y con ellas fin de La Galatea, de quien se está aficionado Vuesa Excelencia». Para interpretar este pasaje, ver Eisenberg.

${ }^{2}$ El texto se conserva en la British Library en una suelta (s.l., s.i., s.a.) que Cotarelo supone edición madrileña de finales del XVII. Cotarelo XXVII.
} 
Por otra parte, en tiempos del rey Mauregato, lejos ya de lo legendario y con unos argumentos que distan mucho de las fuentes épicas o cronísticas tradicionales de los que habían hecho uso Juan de la Cueva, Lope y Cubillo, Mira de Amescua escribió Las desgracias del rey Alfonso el Casto en 1616. Pero hay otros autores que se interesaron por la figura del héroe como Lope de Liaño y su Bernardo del Carpio en Francia. En esta ocasión, de nuevo muy lejos de la figura del legendario Bernardo hispánico, ambientada en Francia en la época del rey franco Ludovico Pío, su argumento gira en torno a la liberación, por parte de Bernardo, de un rey encarcelado por sus hijos.

Mi propósito en las líneas que siguen es esbozar, sin mayores precisiones por ahora, un panorama muy general del estado de la cuestión que nos sirva como marco preliminar para adentrarme después en los vericuetos escriturarios de la comedia que Cubillo dedicó al héroe español El conde de Saldaña $y$ hechos de Bernardo del Carpio, que no es sino la segunda parte de su Conde de Saldaña.

A juzgar por los datos que poseemos, las dos comedias del dramaturgo Álvaro Cubillo de Aragón fueron las más exitosas y las de mayor calidad dramática sobre la materia de Bernardo del Carpio dentro de todo el teatro aurisecular. Este hecho, unido a la valoración general de la crítica respecto a sus dos partes, vienen avalados siempre por el supuesto éxito que alcanzaron dichas piezas en los escenarios (a tenor siempre de lo resbaladizo de las noticias que se conservan de ellas) (ver Cubillo, ed. Lázaro, 2015, 78-86), por la cantidad de ediciones registradas a lo largo de los siglos XVII y XVIII (ver Cubillo, ed. Lázaro, 2017, 27-40), y aun por los compiladores y creadores del canon del mejor teatro áureo del siglo XIX.

Antes de incidir en detalles más precisos sobre su escritura y contextualización, cabe hacer una reseña preliminar de la fortuna crítica de la obra. De entrada, es necesario dejar constancia sobre la escasa benevolencia de la mayoría de la crítica con respecto a esta continuación, que veía muy alejada en cuanto a logros estéticos con respecto a la primera, que fue considerada incluso muy superior a la versión de Lope (ver Lista 290; Mesonero Romanos 98; Barrera y Leyrado 113; Schack 379-382; Schaeffer 90-105; Menéndez Pelayo, «Estudio preliminar» a Obras de Lope de Vega, XVI, 125-126). La raíz de estos juicios poco halagüeños hay que buscarla en los comentarios pioneros de Menéndez Pelayo a partir del análisis un tanto desafortunado de su supuesta fuente El casamiento en la muerte. En ellos Menéndez Pelayo la consideraba «una obra mucho más arreglada que la de Lope, pero sumamente inferior a ella en savia tradicional, en pasión y en movimiento» (Menén- 
dez Pelayo, «Estudio preliminar» a Obras de Lope de Vega, XVI, 143). A partir de ese momento, casi todos los estudiosos continuaron cultivando una suerte de prejuicio estético heredado de la crítica decimonónica, y se limitaron a repetir de modo irreflexivo su superficialidad, ${ }^{3}$ a tacharla de «muy inferior» (Cotarelo y Mori 252) o a compararla, como hizo Whitaker, como patente demérito artístico con respecto a la primera: «In artistic merit Cubi1lo's play in no way competes with its model» (Whitaker 37). Y olvidaron por completo en mi opinión que solo si se obvia la influencia lopesca, se puede dar un sentido completo a las dos partes de El conde de Saldaña. Únicamente de este modo se puede entender la coherencia entre ambas, la intencionalidad del autor y la particular preceptiva que acompañó su escritura. No obstante, he de señalar que fueron los hispanistas alemanes, que valoraron y analizaron más detenidamente la dramaturgia de Álvaro Cubillo, quienes se alejaron de esta crítica basada en prejuicios realmente acientíficos. En primer lugar, destaca Adolf Friedrich von Schak, para quien El conde de Salda$\tilde{n} a$ se encuentra entre las más meritorias del granadino (Schack 379-382), y cuyas dos partes constituyen «quizá la mejor obra dramática que trata de la historia de Bernardo del Carpio y la que se ha sostenido más largo tiempo en el teatro» (Schack 382). ${ }^{4}$ Por su parte, Adolf Schaeffer (90-105) destacó de la segunda parte el momento:

cuando Bernardo está contemplando la estatua de su padre sobre aquella tumba, de repente, la estatua deja caer su bastón delante de los pies del hijo heroico para indicarle que él será el heredero digno de su padre. (Schaeffer 102) ${ }^{5}$

\footnotetext{
${ }^{3}$ Ver Valbuena Prat, «Introducción» a su edición de Las muñecas de Marcela. El Señor de Noches Buenas LXXVIII. En mi opinión, se hace necesario señalar ciertas contradicciones subyacentes entre los criterios estéticos aplicados por Menéndez Pelayo y Valbuena Prat para el enjuiciamiento crítico de El conde de Saldaña y Hechos de Bernardo del Carpio de Cubillo en comparación con los modelos lopescos, así como una discutible actitud pacata o excesivamente escrupulosa que Valbuena, sobre todo, deja entrever cuando afirma que «no se puede juzgar el valiente esbozo de Lope, por el plan perfecto de la obra del granadino; ni la ternura de este, por la bárbara grandeza del creador de nuestro teatro» («Introducción» LXXV).

${ }^{4}$ Traducción de la autora. «Vielleicht die beste behandlung der geschichte des Bernardo de Carpio und diejenige, welche sich am längsten auf der Bühne behauptet hat».

${ }^{5}$ Traducción de la autora. «Ein schöner Zug in diesem zweiten Theile ist folgender. Als Bernardo vor dem Grabmonument mit der Bildsäule seines Vaters in Betrachtung versunken dasteht, lässt die Statue den Feldherrnstab in ihrer Hand zu den Füssen des heldenmüthigen Sohnes fallen, damit andeutend, dass das Zeichen, welches den Vater stets zum Ruhme geführt, in dem Sohne einen würdigen Erben gefunden habe».
} 
Pasemos ya a analizar con detalle el proceso de escritura de la segunda parte de El conde de Saldaña que lleva por título El conde de Saldaña y hechos de Bernardo del Carpio. Se trata de un proceso de autoreescritura dramática o refundición de una obra propia anterior que a su vez toma como fuente una obra primitiva de Lope de Vega, El casamiento en la muerte (aunque muy parcialmente). Como se verá a continuación, se trata de dos obras que, a pesar de llevar por título el mismo nombre, presentan cambios de suficiente entidad como para considerarlas diferentes y no variantes de la misma obra, aunque sí versan sobre la misma materia. Esta técnica de reutilización de materiales formaba parte de la praxis compositiva de Cubillo (y de otros dramaturgos de la época) y se encaminaba siempre hacia la supuesta obtención de una mayor eficacia dramática y muy probablemente a una mayor difusión escénica y editorial.

Comencemos, pues, por su versión manuscrita que consta de dos testimonios que abrevio como $M s_{1}$ y $M s_{2}$.

El manuscrito $M s_{1}$, con letra del siglo XVII, consta de 55 hojas en $4^{\circ}(23 \times$ $17 \mathrm{~cm}$.), numeradas de la 1 a la 53 (con numeración duplicada en las hojas de la tercera jornada, la sucesiva general del manuscrito y la particular de la 1 a la 18). La copia se llevó a cabo al menos por dos manos distintas: una se ocupó de la primera jornada y de la tercera, y, la otra, de la segunda jornada. De escritura impecable, se observa una sola tachadura en la hoja 16r (correspondiente a los vv. 652-653).

El manuscrito $M s_{2}$, con letra de finales del siglo XVII, consta de 54 hojas en $4^{\circ}(21 \times 15 \mathrm{~cm}$.), numeradas de la 1 a la 54 . La copia ha sido realizada, al menos, por dos manos distintas: la primera corresponde a la primera jornada y la segunda, a las jornadas segunda y tercera. Como en el manuscrito $M s_{1}$, se trata de una copia de escritura impecable, con un par de lapsus calami uno en el v. 1630 y otro en el v. 1728.

La versión impresa, por su parte, apareció por primera vez en una Parte trece de comedias varias, integrada por una colección de doce comedias y varios entremeses y loas pertenecientes a diversos autores, titulada De las mejores el mejor libro nuevo de comedias varias, nunca impresas, compuestas por los mejores ingenios de España, publicada en Madrid en 1660. ${ }^{6}$ Bien porque no existieran (que probablemente, sí) bien porque no hayan podido

\footnotetext{
${ }^{6}$ Comedia famosa / Segunda parte del Conde de Saldaña y hechos de / Bernardo del Carpio. / De Álvaro Cubillo de Aragón, en De los mejores el mejor libro nvevo de comedias varias, nvnca impressas, compuestas por los mejores ingenios de España. Parte treze, Madrid, Mateo Fernandez, 1660, 42-72 [Biblioteca Nacional de España, Sign. Ti/16 (13)].
} 
ser conservados o localizados, no contamos con otros testimonios de la transmisión textual durante el siglo XVII. No poseemos ningún dato de carácter externo o interno que nos permita fechar con exactitud su composición. Las copias manuscritas de esta versión no presentan aprobaciones o censuras como sucedía con la primera parte que permitían datar con cierta precisión las mismas. Ahora bien, podemos suponer que su composición no debió de retrasarse mucho tiempo con respecto a la de la primera parte, por lo que es plausible que Cubillo pudo componerla poco después de la primera y, en todo caso, en la década -quizá a mediados- de los años 40, dado que su temática y su intención dramática remite sin duda a los enfrentamientos bélicos y a la resistencia de la monarquía española frente a las aspiraciones hegemónicas de Francia.

La fortuna editorial del texto dramático corrió mejor suerte en el siglo XVIII ya que se conservan un buen número de ediciones sueltas. Hasta un total de catorce testimonios han podido ser localizados por el momento: una edición salida de las prensas murcianas de Juan López, otra de la Imprenta Real de Sevilla, tres de la imprenta madrileña de Antonio Sanz, otras tres de las prensas salmantinas de la Santa Cruz, dos de la imprenta valenciana de los Orga, una de la imprenta burgalesa de la Santa Iglesia, otra de la prensa barcelonesa de Juan Serra y Nadal, y dos últimas de las imprentas madrileñas de Quiroga e Isidro López. Las imprentas del siglo XIX acogieron El conde de Saldaña y hechos de Bernardo del Carpio en diversas ocasiones. Está localizada una edición valenciana de la imprenta de Ildefonso Mompié (1822), otra en La Habana, salida de la imprenta de R. Oliva (1840) y, finalmente, la edición de Mesonero Romanos incluida en su colección titulada Dramáticos posteriores a Lope de Vega (Madrid, Rivadeneira, 1858).

A continuación analizaremos las fuentes de las que se nutre Cubillo para escribir las dos partes de El conde de Saldaña.

En primer lugar, fue Menéndez Pelayo quien aseveró que Las mocedades de Bernardo del Carpio y El casamiento en la muerte de Lope de Vega son las dos obras sobre la materia de Bernardo del Carpio fuente de inspiración de las de Cubillo y, aunque no fue tanta su influencia (sobre todo en la segunda parte), se puede decir que los dos dramaturgos se nutrieron de las mismas fuentes y siguieron un mismo patrón. Buena parte de los motivos proceden de la Primera Crónica General ${ }^{7}$ y del romancero. No cabe duda de que había sido el romancero el encargado de mantener viva la materia de Bernardo. Agustín Durán en El Romancero General (1849) compila 46 ro-

\footnotetext{
${ }^{7}$ Ver capítulo 619.
} 
mances que se refieren a dicha materia, de los que cabe destacar para esta segunda parte: sus hazañas caballerescas, su oposición a las pretensiones anexionistas de Carlomagno, los episodios relativos a la batalla de Roncesvalles, sus enfrentamientos con los moros o los desplantes reales. ${ }^{8}$ Dicha antología procedía de las diversas colecciones romanceriles del siglo XVI: el Cancionero de Romances de Martín Nucio, 1550; los Romances de Lorenzo de Sepúlveda, 1551; la Rosa de romances de Juan de Timoneda, 1561; el Romancero de Alonso de Fuentes, 1579, etc., cuyo ciclo dedicado al conde de Saldaña y Bernardo del Carpio se trasladó en buena parte al Romancero General de $1600 .^{9}$

Un poco más en detalle, la comedia de Lope se sirvió de la figura de un Bernardo hispánico (ver Gómez Redondo 1295-1298). Como ya advirtió Lozano «la relación genealógica con Carlomagno desaparece de las dos comedias de Lope; emparentar a Bernardo con los franceses privaría al público del elemento nacionalista y patriótico» (ver Lozano 92-93). Así pues, el dramaturgo granadino habría partido de Las mocedades de Bernardo del Carpio para escribir El bastardo de Castilla, que posteriormente reescribiría creando la versión definitiva de El conde de Saldaña (ver Lázaro 64-68); y para la segunda parte, El conde de Saldaña y hechos de Bernardo del Carpio, Cubillo habría tomado una pequeña parte del argumento de El casamiento en la muerte.

Si bien esto último es cierto, en la segunda parte no se observa tanta influencia de Lope como en la primera. Se puede decir que Cubillo tomó el marco de la batalla de Roncesvalles que sirve de argumento a El casamiento en la muerte y lo desarrolló, al igual que había hecho en su primera parte, prescindiendo de todos aquellos episodios que no enriquecían la figura de Bernardo del Carpio. Su objetivo era claro, quería mostrar al paladín como símbolo de la resistencia española frente a los franceses y engrandecer su figura como el héroe que acabó con la vida de Roldán. Básicamente quiso constatar frente al pueblo la valentía y arrojo del ejército español, de su hostilidad perpetua con los franceses, sin olvidar la inestable enemistad con los moros que en ocasiones pasaban a ser sus aliados.

No obstante, en ambas obras aparecen algunas secuencias y motivos semejantes. Una de estas secuencias es la embajada de Bernardo en París para pedir a Carlomagno que olvide las promesas del rey español, donde se pro-

\footnotetext{
${ }^{8}$ Romancero general 417-439.

${ }^{9}$ Las diversas ediciones y antologías romanceriles más o menos modernas han seguido recogiendo buena parte de los romances del ciclo de Bernardo: Primavera y flor de romances 26-47; Flor nueva de Romances Viejos 69-102; El Romancero Viejo 118-121; Romancero 180-185; Romancero 97-105.
} 
ducen acaloradas discusiones sobre el patriotismo y los retos de Bernardo ante los paladines franceses. Otra, la preparación y desarrollo de la batalla de Roncesvalles: en ambas obras los españoles luchan contra los franceses, apoyados por el ejército de Marsilio comandado por el moro Bravonel, donde salen triunfantes.

Quizá uno de los rasgos más destacados sea la supresión de aquellos capítulos donde hay un exceso de atmósfera épica. Cubillo trata de atemperarlos (en ocasiones aumentado la extensión de las secuencias) y de obtener un mayor ennoblecimiento y humanización del héroe. Esto se puede observar, por ejemplo, en el incremento considerable de la estancia de Bernardo del Carpio en París, que, como resultado, le da oportunidad al héroe español de celebrar una justa de la que sale vencedor. Del mismo modo, Cubillo ensalza las virtudes del héroe y del antihéroe -Roldán y Bernardo-: muestra la lealtad de Bernardo al devolver al criado Pierres con vida para hacer partícipe a Roldán de los planes de Ganelón, que se corresponde con la del paladín francés que, para mostrar su agradecimiento, libera al escuadrón de mujeres españolas que habían acudido al campo de batalla y las escolta hasta su campamento.

La versión cubillesca presenta episodios novedosos que se corresponden con la primera parte (El conde de Saldaña): Bernardo acaba con la vida de don Rubio en defensa propia, se acentúa la figura de la amada de Bernardo, doña Sol, se da continuidad a la figura del gracioso de Monzón y, por unos momentos, se da vida al mismísimo conde de Saldaña. Se introduce la figura de un soldado portugués que, aunque forma parte del ejército francés, ensalza la misión española.

En esta ocasión, el autor granadino compone una obra muy lejana a las de Lope pues casi no utiliza el tema de la batalla de Roncesvalles. Se caracteriza la versión de Cubillo por el delicado tratamiento que da al tema amoroso y por suprimir, como veremos a continuación, aquellas escenas épicas superfluas que no cumplían con el objetivo de que se desviaban del objetivo de ensalzar y ennoblecer al héroe Bernardo del Carpio.

Tanto en la versión manuscrita como en la impresa encontramos una serie de cambios sustanciales entre la «escritura» y su «reescritura», siendo la segunda más cercana a la preceptiva teatral del propio Cubillo, quien insistía en la idea de que: «No es desahogo ver en la comedia / el insulto, el agravio, la tragedia / el blasfemo de Dios amenazado, / el duelo ejecutado, / la virtud ofendida, / y a precio de una vida y otra vida / con bárbara violencia, / la traición, la maldad y la insolencia» (Cubillo de Aragón, El enano de los Musas, «Prólogo al lector»). 
Siguiendo lo expuesto en los párrafos precedentes, ofrezco a continuación algunos ejemplos más de los cambios más notables en el proceso de autoreescritura, referidos en concreto a la secuencia episódica, la modificación y supresión de personajes, y el ambiente donde se desarrolla la obra y su extensión.

Desde la perspectiva de la secuencia episódica, los cambios operados en ella no modifican en exceso el argumento ya que se mantiene la misma estructura y desarrollo argumental con algunas variaciones menores. Dichas variaciones obedecen a dos razones: por un lado, al intento de no restar efectismo dramático y, por otro, a la intencionalidad de elevar la categoría del protagonista; para ello, se eliminan de la versión impresa aquellos episodios de tono épico existentes en la versión manuscrita. De este modo, el enfrentamiento de Bernardo del Carpio con Oliveros se suprime y directamente la lucha tiene lugar con Roldán.

En cuanto a la modificación del elenco de personajes, la modificación más radical guarda relación con el criado Monzón. Cubillo de Aragón, siguiendo su preceptiva teatral, trata de atemperarlo y suaviza mucho sus formas, así, mientras que en la versión manuscrita el criado ridiculizaba constantemente al enemigo, en la versión impresa se atempera y su función de gracioso se dirige a contrarrestar con su espíritu jocoso los efectos dramáticos de la obra. El personaje de Tancredo también modera sus formas y, mientras en la versión manuscrita aparece perfilado como un personaje intransigente y molesto, en la versión impresa se reviste de cualidades caballerescas. Por otra parte, el personaje del soldado portugués existente en la versión manuscrita desaparece en la versión impresa, y con su supresión también se elimina su motivo argumental, que resultaba de escasa importancia para el desarrollo de la trama. También desciende el número de paladines que luchan con Bernardo o que forman parte de la batalla.

En lo referente al ambiente, una de las diferencias más radicales tiene que ver con el ambiente belicoso ya que, se apacigua o suprime en la versión impresa. Como acabamos de señalar, desciende el número de personajes que forman parte del conflicto bélico, se reduce la estrategia militar y se simplifica el desarrollo de la campaña. Sin embargo, el dramaturgo granadino dibuja un cuadro conformado por un escuadrón de mujeres guerreras que pasaba desapercibido en la versión manuscrita. Y, como era de esperar, suprime de la versión impresa la aparición con carácter premonitorio del difunto conde de Saldaña, con total probabilidad, por su falta de verosimilitud. 
Por último, en relación a la extensión del texto, el número de versos que componen estas dos versiones es muy similar e inferior a las dos versiones que conforman la primera parte. La versión manuscrita se compone de 2397 versos y la versión impresa cuenta con 2286 versos. Si comparamos estos datos con los de la primera parte, observamos que el manuscrito de El bastardo de Castilla cuenta con 479 versos más y que el impreso de El conde de Saldaña cuenta con 661 versos más (ver Lázaro 59-74).

\section{EL PROCESO DE REESCRITURA}

Para poder realizar un estudio contrastado entre el proceso de escritura (manuscrito) y su reescritura (impreso) se ha realizado un estudio en el que se ha cotejado cada uno de los versos del impreso con los del manuscrito. En cada una de las tres jornadas, de modo independiente se ha tenido en cuenta si la reescritura era parcial (dentro de un verso), de nuevos versos por adición, o incluso de nuevos parlamentos. Con todo ello se han elaborado las tablas y los gráficos que siguen:

Primera Jornada

\begin{tabular}{|c|c|c|c|c|c|}
\hline \multirow{2}{*}{$\begin{array}{c}\text { Reescritura Completa (245 vv.) } \\
36,03 \%\end{array}$} & \multicolumn{4}{|c|}{$63,97 \%$} \\
\hline Directo & $\%$ & Con Variante & $\%$ & Idénticos & $\%$ \\
\hline 245 & $36,03 \%$ & 50 & $7,53 \%$ & 385 & $56,62 \%$ \\
\hline Total & \multicolumn{5}{|c|}{680} \\
\hline
\end{tabular}

SEgunda Jornada

\begin{tabular}{|c|c|c|c|c|c|}
\hline \multicolumn{2}{|c|}{$\begin{array}{c}\text { ReEscritura Completa (210 vv.) } \\
22,83 \%\end{array}$} & \multicolumn{4}{|c|}{$77,17 \%$} \\
\hline Directo & $\%$ & Con Variante & $\%$ & Idénticos & $\%$ \\
\hline 210 & $22,83 \%$ & 55 & $5,98 \%$ & 655 & $71,1 \%$ \\
\hline Total & \multicolumn{5}{|c|}{} \\
\hline
\end{tabular}


TERCERA JORNADA

\begin{tabular}{|c|c|c|c|c|c|}
\hline \multicolumn{2}{|c|}{$\begin{array}{c}4 \\
\text { Reescritura completa (424 vv.) } \\
53,20 \%\end{array}$} & \multicolumn{4}{|c|}{$\mathbf{4 6 , 8 0 \%}$} \\
\hline Directo & $\%$ & Con Variante & $\%$ & Idénticos & $\%$ \\
\hline 424 & $53,20 \%$ & 92 & $11,54 \%$ & 281 & $35,26 \%$ \\
\hline Total & \multicolumn{5}{|c|}{797} \\
\hline
\end{tabular}

Cómputo global

\begin{tabular}{|c|c|c|c|c|c|}
\hline \multirow{2}{*}{$\begin{array}{c}\text { ReEscritura Completa (879 vv.) } \\
\text { 36,67\% }\end{array}$} & \multicolumn{4}{|c|}{ Retenidos (1518 vv.) } \\
$63,33 \%$ \\
\hline Directo & $\%$ & Con Variante & $\%$ & Idénticos & $\%$ \\
\hline 879 & $36,67 \%$ & 197 & $8,22 \%$ & 1321 & $55,11 \%$ \\
\hline Total & \multicolumn{5}{|c|}{2397} \\
\hline
\end{tabular}

Si pasamos a contrastar los datos procedentes de los gráficos correspondientes a la reescritura, vemos que en las tres jornadas se observan casos de reescritura parcial, de nuevos parlamentos. Hay aproximadamente un $6 \%$ de versos con reescritura parcial del total de versos en la primera jornada; un $7,5 \%$ de versos en la segunda jornada y un $11,5 \%$ de versos en la tercera jornada. Es precisamente esta última jornada la que ha sido sometida a un proceso de reescritura más integral, pues es donde Cubillo modifica levemente las intervenciones, aminorando los elementos belicosos e introduciendo la figura femenina en el campo de batalla.

En la primera jornada el proceso de reescritura afecta aproximadamente a un $23 \%$ de versos nuevos; en la segunda jornada, a un $36 \%$ de versos nuevos, mientras que en la tercera jornada la reescritura alcanza el $53 \%$ de versos nuevos.

De todo este análisis pormenorizado podemos concluir que el dramaturgo granadino Cubillo de Aragón, tomando como punto de partida el manuscrito de El conde de Saldaña y hechos de Bernardo del Carpio, en un proceso de auto-reescritura, lo reescribió y creó la versión impresa con el mismo nombre, 
que conserva un $55,11 \%$ de los versos originales, donde aparecen modificaciones con una reescritura parcial en el $8,22 \%$ de los versos y donde se presentan hasta un total de $36,67 \%$ de versos nuevos, es decir, de su reescritura total.

Todo este proceso de mejora al que somete el texto, desde los cambios estructurales hasta la supresión de personajes, son tan solo un fiel reflejo de su estilo, muy cercano al calderoniano, que se aleja de la hinchazón retórica y de los excesos tremendistas, con una tendencia a la atemperación.

En conclusión, el uso que hace el dramaturgo de la materia legendariopatriótica de Bernardo del Carpio no dista mucho de los intereses habituales en el teatro áureo, cuyo objetivo no era otro que exaltar la monarquía austríaca y avivar el espíritu patriótico, en un momento clave de la necesidad de enfatizar la tenacidad y resistencia de la monarquía española frente a las pretensiones hegemónicas francesas.

En esta ocasión, y dando continuidad a la primera parte de El conde de Saldaña, Cubillo se nutre de las obras de Lope de Vega (Las Mocedades de Bernardo del Carpio y El casamiento en la muerte) reutilizando sus materiales para sus nuevas versiones, refundiéndolos y reescribiéndolos, en respuesta siempre a los designios constructivos de su propia poética, y cómo no, seguramente plegándose a la caprichosa tiranía de los espectadores de su tiempo.

\section{TRABAJOS CITADOS}

Antonucci, Fausta. "La materia caballeresca en el primer Lope de Vega". La comedia de caballerías. Actas de las XXVIII Jornadas de teatro clásico, Almagro, julio de 2005, edited by Felipe B. Pedraza Jiménez, Rafael González Cañal y Elena Marcello, Universidad de Castilla-La Mancha / Festival de Almagro, 2006, pp. 59-77.

Barrera y Leyrado, Cayetano Alberto de la. Catálogo Bibliográfico y Biográfico del Teatro Antiguo Español desde sus orígenes hasta mediados del siglo XVIII (Madrid, 1860). Tamesis, 1968.

Cotarelo y Mori, Emilio. «Dramáticos españoles del siglo XVII. Álvaro Cubillo de Aragón». Boletín de la Real Academia Española, vol. 5, 1918, pp. 3-23 y 241-280.

Cubillo de Aragón, Álvaro. El enano de los Musas. Comedias y obras diversas, con un poema de las Cortes del León y del Águila, acerca del búho gallego (Madrid, María de Quiñones, 1654). Georg Olms Verlag, 1971.

Cubillo de Aragón, Álvaro. Las muñecas de Marcela. El Señor de Noches Buenas, edited by Ángel Valbuena Prat, Compañía Ibero-Americana de Publicaciones, 1928.

Cubillo de Aragón, Álvaro. El conde de Saldaña, edited by Rebeca Lázaro Niso, Academia del Hispanismo, 2015. 
Cubillo de Aragón, Álvaro. El conde de Saldaña y hechos de Bernardo del Carpio, edited by Rebeca Lázaro Niso, Academia del Hispanismo, 2017.

Cueva, Juan de. "Comedia de la libertad de España por Bernardo del Carpio". Teatro renacentista, edited by Miguel Ángel Pérez Priego, Plaza-Janés, 1987.

Chevalier, Maurice, L'Arioste en Espagne, Institut d'Etudes Ibériques et Ibéro-Américaines de l'Université de Bordeaux, 1966.

Eisenberg, Daniel. La interpretación cervantina del Quijote. Compañía literaria, 1995.

Flor nueva de Romances Viejos, edited by Ramón Menéndez Pidal, Espasa Calpe, 1978.

Gómez Redondo, Fernando. "Bernardo del Carpio". Gran enciclopedia cervantina, II, Castalia, 2006, pp. 1295-1298.

Lázaro Niso, Rebeca. "La leyenda de Bernardo del Carpio y su proyección en la literatura." Cuadernos de Aleph, vol. 7, 2015, pp. 79-95.

Lázaro Niso, Rebeca. "Sobre la reescritura dramática en el Siglo de Oro: el caso de El bastardo de Castilla." Roczniki Humanistyczne vol. 66, fasc. 5, 2018, pp. 59-73.

Lista, Alberto. Lecciones de literatura española, II. José Repullés, 1853.

Lozano, Joaquin Roses. "Algunas consideraciones sobre la leyenda de Bernardo del Carpio en el teatro de Lope de Vega." Inti: Revista de literatura hispánica, vol. 1, no. 28, 1988, pp. 88-105.

Mesonero Romanos, Ramón de. "Teatro de Cubillo.” Semanario Pintoresco Español. 1852, pp. 97-100.

Morley, Sylvanus Griswold, and Courtney Bruerton. Cronología de las comedias de Lope de Vega. Gredos, 1968.

Obras de Lope de Vega, XVI. Crónicas y leyendas dramáticas españolas, edited by Marcelino Menéndez Pelayo, Atlas, 1966.

Primavera y flor de romances vol. I, edited by Fernando José Wolf and Conrado Hofmann, Asher, 1856.

Primera Crónica General de España vol. II, edited by Ramón Menéndez Pidal, Gredos, 1977.

Ratcliffe, Marjorie. "Honor y legitimidad: Bernardo del Carpio en el Siglo de Oro." Edad de oro cantabrigense: actas del VII Congreso de la Asociación Internacional de Hispanistas del Siglo de Oro, edited by Anthony J. Close and Sandra María Fernández Vales, Robinson College, 2006, pp. 59-74.

Rey Hazas, Antonio, and Florencia Sevilla Arroyo. "La casa de los celos." Gran enciclopedia cervantina, III, edited by Carlos Alvar, Castalia, 2006, pp. 1958-1966.

Romancero Castellano [Cancionero de romances, Amberes: 1550], edited by Carlos Clavería, Castro, 2004.

Romancero general o Colección de romances castellanos anteriores al siglo XVIII, Tomo Primero, edited by Agustín Durán, Rivadeneira, 1849.

Romancero general o Colección de romances castellanos anteriores al siglo XVIII, Tomo Segundo, edited by Agustín Durán, Rivadeneira, 1851.

Romancero viejo, El, edited by Mercedes Díaz Roig, Cátedra, 1976.

Romancero, edited by Pedro M. Piñero Ramírez, Biblioteca Nueva, 1999.

Romancero, edited by Paloma Díaz-Masm, Crítica, 2006. 
Schack, Adolf Friedrich von. Geschichte der dramatischen Literatur und Kunst in Spanien, III. Georg Olms, 1975.

Schack, Adolf Friedrich von. Historia de la literatura y del arte dramático en España, V, Imprenta y Fundición de M. Tello, 1887.

Schaeffer, Adolf. Geschichte des Spanischen Nationaldramas, II (Die periode Calderón's). Brockhaus, 1890.

Vega Carpio, Lope Félix de. "Las mocedades de Bernardo del Carpio." Obras de Lope de Vega XVII. Atlas, 1966, pp. 1-48.

Vega Carpio, Lope Félix de. "El casamiento en la muerte." Comedias de Lope de Vega, Parte I, vol. 2, edited by Luigi Giuliani, Milenio, 1997, pp. 1151-1276.

Watson, Anthony. Juan de la Cueva and the Portuguese Succession. Tamesis, 1971.

Whitaker, Shirley B. The dramatic Works of Álvaro Cubillo de Aragón. North Carolina Studies in the Romance Languages and Literatures. University of North Carolina Press, 1975.

\section{LEKTURY I RESKRYBOWANIA \\ HECHOS DE BERNARDO DEL CARPIO}

\section{Streszczenie}

Przez cały Złoty Wiek reskrybowanie dzieł dramatycznych staje się powszechną formą twórczości literackiej. Niewielu jest dramaturgów, którzy nie odczuwaliby potrzeby powrotu do dzieł swoich poprzedników z zamiarem ulepszenia lub aktualizacji tekstów wobec zmieniających się upodobań publiczności. To ogólne zjawisko jest zilustrowane w tym artykule poprzez analizę na nowo napisanej i wydanej w dwóch różnych wersjach drukowanych przez Álvaro Cubillo de Aragón sztuki poświęconej historyczno-legendarnej tematyce związanej z postacią Bernardo del Carpio: El conde de Saldaña y hechos de Bernardo del Carpio.

Przekład streszczenia angielskiego Stanisław Sarek

Słowa kluczowe: Złoty Wiek; reskrybowanie; sztuki dramatyczne; Bernardo del Carpio; Cubillo de Aragón; Hechos de Bernardo del Carpio.

\section{READINGS AND REWRITINGS \\ OF HECHOS DE BERNARDO DEL CARPIO}

S u m m a ry

Throughout the Golden Age the rewriting of dramatic works becomes a common form of literary creation. Few are the playwrights who did not feel the need to return to the works of their predecessors with the intention of improving or updating texts against the changing tastes of the public. This general phenomenon is exemplified in this paper through the rewriting in two different printed versions carried out by Álvaro Cubillo de Aragón of a play dedicated to the historical-legendary matter related to the figure of Bernardo del Carpio: El conde de Saldaña y hechos de Bernardo del Carpio.

Key words: Golden Age; rewriting; dramatic plays; Bernardo del Carpio; Cubillo de Aragón; Hechos de Bernardo del Carpio. 